\title{
Publisher Correction: Giant topological Hall effect in correlated oxide thin films
}

Lorenzo Vistoli, Wenbo Wang (iD, Anke Sander, Qiuxiang Zhu, Blai Casals (D), Rafael Cichelero, Agnès Barthélémy, Stéphane Fusil, Gervasi Herranz, Sergio Valencia, Radu Abrudan (1), Eugen Weschke, Kazuki Nakazawa, Hiroshi Kohno, Jacobo Santamaria, Weida Wu (1), Vincent Garcia and Manuel Bibes (1)

Correction to: Nature Physics https://doi.org/10.1038/s41567-018-0307-5 published online 15 October 2018.

In the version of this Letter originally published, Hiroshi Kohno's affiliation was incorrectly listed as Department of Earth and Space Science, Graduate School of Science, Osaka University, Osaka, Japan; it should have been Department of Physics, Nagoya University, Nagoya, Japan. This has been corrected in all versions of the Letter.

Published online: 26 October 2018

https://doi.org/10.1038/s41567-018-0357-8

\section{Author Correction: Low-temperature anomaly in disordered superconductors near Bc2 as a vortex-glass property}

Benjamin Sacépé, Johanna Seidemann, Frédéric Gay, Kevin Davenport, Andrey Rogachev (1), Maoz Ovadia, Karen Michaeli and Mikhail V. Feigel'man

Correction to: Nature Physics https://doi.org/10.1038/s41567-018-0294-6 published online 8 October 2018.

In the version of this Article originally published, equation (5) incorrectly said $j_{\mathrm{c}}^{\mathrm{GL}}(B)=\frac{8 e}{3 \sqrt{3} \pi h \rho_{\mathrm{s}}(B) \xi_{\mathrm{GL}}(B)}$; it should have said $j_{\mathrm{c}}^{\mathrm{GL}}(B)=\frac{4 e \rho_{\mathrm{s}}(B)}{3 \sqrt{3} \hbar \xi_{\mathrm{GL}}(B)}$. This has been corrected in all versions of the Article.

Published online: 30 October 2018

https://doi.org/10.1038/s41567-018-0359-6 